\title{
Experiências de práticas de cuidado farmacêutico para pessoas surdas: uma revisão integrativa da literatura
}

\author{
Experiences of pharmaceutical care practices for deaf people: an integrative literature review \\ Experiencias de prácticas de servicios farmacéuticos para personas sordas: una revisión \\ integradora de la literatura
}

Recebido: 10/12/2021 | Revisado: 16/12/2021 | Aceito: 20/12/2021 | Publicado: 03/01/2022

\author{
Kamila Couto de Paula \\ ORCID: https://orcid.org/0000-0003-2044-5333 \\ Hospital do Câncer III, Brasil \\ E-mail: kamilacouto@id.uff.br \\ Gildete da Silva Amorim Mendes Francisco \\ ORCID: https://orcid.org/0000-0001-5185-2092 \\ Universidade Federal Fluminense, Brasil \\ E-mail: gildeteamorin@yahoo.com.br \\ Tatiane Militão de Sá \\ ORCID: https://orcid.org/0000-0001-5675-6171 \\ Universidade Federal Fluminense, Brasil \\ E-mail: tatimili2@yahoo.com.br \\ Elaine Silva Miranda \\ ORCID: https://orcid.org/0000-0002-6204-5023 \\ Universidade Federal Fluminense, Brasil \\ E-mail: elainemiranda@id.uff.br
}

\begin{abstract}
Resumo
Educação no contexto da promoção e recuperação da saúde e prevenção de doenças é uma prática importante e contribui para a compreensão do paciente surdo sobre o seu processo de saúde-doença-cuidado. O objetivo dessa revisão é identificar experiências de inclusão envolvendo pessoas com surdez no âmbito das práticas de cuidados farmacêuticos. Para o desenvolvimento, considerou-se analisar as publicações indexadas de cinco bases de dados, LILACS, Web of Science, Scopus, Embase e Pubmed. As combinações dos descritores constituíram-se pelos operadores booleanos AND e OR: ((Deaf OR Deafness OR "Hearing loss" [All Fields]) AND (Pharmaceutical Services [All Fields] OR Pharmaceutical Care [All Fields])) e ((Deaf OR Deafness OR "Hearing loss" [All Fields]) AND ("Pharmaceutical Services" [All Fields] OR "Pharmaceutical Care" [All Fields])). Foram analisados os artigos nos idiomas inglês, português e espanhol, no período de janeiro de 2011 a setembro de 2021. Os resultados das buscas contemplaram estudos do tipo transversal, qualitativo e quali-quantitativo. Os achados apontaram lacunas no que tange às experiências de farmacêuticos e/ou alunos de graduação em Farmácia sobre educação em saúde para surdos, onze artigos destacam sobre os serviços farmacêuticos. A barreira linguística é um desafio, que necessita ser superado, para que o paciente surdo consiga adquirir informações necessárias sobre a utilização de medicamentos e educação em saúde. Podemos concluir que existem estudos relevantes sobre a oferta e promoção de cuidados farmacêuticos para pessoas surdas, sendo essas estabelecidas em diferentes cenários.
\end{abstract}

Palavras-chave: Cuidados farmacêuticos; Surdez; Língua de sinais; Comunicação em saúde.

\begin{abstract}
Education in the context of health promotion and recovery and disease prevention is an important practice and contributes to deaf patients' understanding of their health-disease-care process. The objective of this review is to identify inclusion experiences involving people with deafness in the scope of pharmaceutical services practices. For development, it was considered to analyze the indexed publications of five databases, LILACS, Web of Science, Scopus, Embase and Pubmed. The combinations of descriptors were constituted by the Boolean operators AND and OR: ((Deaf OR Deafness OR "Hearing loss" [All Fields]) AND (Pharmaceutical Services [All Fields] OR Pharmaceutical Care [All Fields])) and ((Deaf) OR Deafness OR "Hearing loss" [All Fields]) AND ("Pharmaceutical Services" [All Fields] OR "Pharmaceutical Care" [All Fields])). Articles in English, Portuguese and Spanish were analyzed, from January 2011 to September 2021. The search results included cross-sectional, qualitative and quali-quantitative studies. The findings pointed out gaps with regard to the experiences of pharmacists and/or undergraduate pharmacy students on health education for the deaf, eleven articles stand out about pharmaceutical services. The language barrier is a challenge that needs to be overcome, so that the deaf patient can acquire necessary information about the use of medication and health
\end{abstract}


education. We can conclude that there are relevant studies on the offer and promotion of pharmaceutical care for deaf people these being established in different settings.

Keywords: Pharmaceutical services; Deafness; Sign language; Health communication.

\section{Resumen}

La educación en el contexto de la promoción de la salud y la recuperación y prevención de enfermedades es una práctica importante y contribuye a que los pacientes sordos comprendan su proceso salud-enfermedad-atención. El objetivo de esta revisión es identificar experiencias de inclusión que involucren a personas con sordera en el ámbito de las prácticas de servicios farmacéuticos. Para su desarrollo se consideró analizar las publicaciones indexadas de cinco bases de datos, LILACS, Web of Science, Scopus, Embase y Pubmed. Las combinaciones de descriptores fueron constituidas por los operadores booleanos Y y O: ((Sordera O Sordera O "Pérdida de audición" [Todos los campos]) Y (Servicios farmacéuticos [Todos los campos] O Atención farmacéutica [Todos los campos])) y ((Sordos) O Sordera O "Pérdida de audición" [Todos los campos]) Y ("Servicios farmacéuticos" [Todos los campos] O "Atención farmacéutica" [Todos los campos])). Se analizaron artículos en inglés, portugués y español, desde enero de 2011 hasta septiembre de 2021. Los resultados de la búsqueda incluyeron estudios transversales, cualitativos y cuali-cuantitativos. Los hallazgos señalaron lagunas con respecto a las experiencias de los farmacéuticos y /o estudiantes de pregrado de farmacia en educación en salud para sordos, destacan once artículos sobre servicios farmacéuticos. La barrera del idioma es un desafío que debe superarse para que el paciente sordo pueda adquirir la información necesaria sobre el uso de medicamentos y educación sanitaria. Podemos concluir que existen estudios relevantes sobre la oferta y promoción de la atención farmacéutica para las personas sordas, que se están implantando en diferentes contextos.

Palabras clave: Servicios farmacéuticos; Sordera; Lengua de signos; Comunicación en salud.

\section{Introdução}

Além das malformações que levam à surdez desde o nascimento, a perda de audição pode ser desencadeada por diversos fatores tais como infecções, causas relacionadas ao parto e uso de medicamentos ototóxicos (WHO, 2020). No Brasil, segundo o Instituto Brasileiro de Geografia e Estatística (IBGE), ao redor de 10 milhões de pessoas são surdas (IBGE, 2020). Esta população é, historicamente, estigmatizada e por este motivo, muitas vezes evitam procurar atendimento em geral, inclusive os prestados em serviços em saúde (Hyoguchi et al., 2020).

A comunicação constitui uma abordagem importante de interação entre profissionais de saúde e pacientes no intuito de alcançar melhores desfechos das ações de cuidado (Vinholes, Alano \& Galato, 2009). Entretanto, muitos profissionais de saúde vivenciam diferentes obstáculos quando confrontados com pessoas surdas e vice-versa (Fergunson \& Shan, 2016). No que tange a utilização de medicamentos, a barreira linguística torna-se um desafio expresso na dificuldade de informar sobre o uso seguro, garantir adesão ao tratamento, esclarecer e identificar os efeitos adversos e interações medicamentosas (Hyoguchi, Ohmitsu \& Kubota, 2020). Da mesma forma, ficam comprometidas as ações de educação em saúde voltadas para provocar mudanças positivas de cuidado em diferentes comunidades (Soares et al., 2016).

Uma das formas de viabilizar o acesso de pessoas surdas aos estabelecimentos de saúde consiste na identificação e emprego de metodologias ativas e instrumentos educativos que permitam melhorar a comunicação entre o profissional e a pessoa surda (Mathews et al., 2011; Hyoguchi, Ohmitsu \& Kubota, 2020).

A língua de sinais é o meio de transmissão de mensagens que os surdos adquirem em algum momento da vida para poder comunicar-se com outras pessoas surdas, essa língua difere da língua falada pelo fato de ser feita por sinalizações com as mãos e por meio de expressões faciais e as vezes corporais que ajudará o interlocutor a compreender a mensagem que se deseja transmitir. Essas sinalizações devem acontecer em um espaço limitado pelo sinalizador de modo que o interlocutor não tenha dificuldades em entender o que está sendo transmitido (Brasil, 2005).

Estabelecida pela Lei $\mathrm{N}^{\circ} 10.436$ de 24 de abril de 2002, a Língua Brasileira de Sinais (Libras) é reconhecida como meio de comunicação pelas pessoas surdas e assim elas manifestam a sua cultura (Brasil, 2002). Dessa forma, é importante o estímulo aos profissionais de saúde, entre os quais farmacêuticos, no sentido de aprenderem Libras questões sobre cultura surda para assim poderem contribuir na melhoria da qualidade de assistência farmacêutica que essa comunidade necessita. Pelas 
reflexões expostas o objetivo do presente artigo é identificar experiências de inclusão envolvendo pessoas com surdez no âmbito das práticas de cuidados farmacêuticos.

\section{Metodologia}

Trata-se de uma revisão integrativa. Este é um método de revisão amplo que caracteriza-se por analisar as publicações mais relevantes a partir de um tema delimitado previamente, com o intuito de auxiliar profissionais de saúde na tomada de decisão e na melhoria da prática clínica. A revisão integrativa incorpora diferentes metodologias com o objetivo de alcançar uma compreensão particular de um problema relacionado aos cuidados em saúde (Whittmore \& Knafl, 2005).

A busca dos estudos incluídos na revisão foi realizada em cinco bases de dados: Literatura Latino Americana e do Caribe em Ciências da Saúde (LILACS), Web of Science, Scopus, Embase e Pubmed. Considerando os termos de pesquisas mais relevantes que se contextualizam com o tema escolhido, optou-se por descritores indexados no The Medical Subject Headings (MeSH) Database, da National Library of Medicine (Quadro 1).

Quadro 1. Descritores indexados nas bases de dados, The MeSH Database da National Library of Medicine, utilizados na estratégia de busca.

\begin{tabular}{|c|c|}
\hline Descritores em inglês & Descritores em português \\
\hline Deaf & Surdo \\
\hline Deafness & Surdez \\
\hline Hearing loss & Perda auditiva \\
\hline Pharmaceutical Services & Assistência Farmacêutica \\
\hline Pharmaceutical Care & Atenção Farmacêutica \\
\hline
\end{tabular}

Fonte: Autores.

As combinações dos descritores constituíram-se pelos operadores booleanos AND e OR, perfazendo o seguinte desenho de estratégia de recuperação de estudos: ((Deaf OR Deafness OR "Hearing loss" [All Fields]) AND (Pharmaceutical Services [All Fields] OR Pharmaceutical Care [All Fields])), na base de dados Web of Science, Pubmed, Embase e LILACS; ((Deaf OR Deafness OR "Hearing loss" [All Fields]) AND ("Pharmaceutical Services" [All Fields] OR "Pharmaceutical Care" [All Fields])), na base de dados Scopus. Todos os artigos completos, disponíveis para leitura na íntegra, escritos em inglês, português ou espanhol, que trouxeram experiências farmacêuticas com enfoque para a comunidade surda foram considerados nesta revisão. A busca compreendeu o período entre janeiro de 2011 e setembro de 2021.

Foram estabelecidos como critérios para elegibilidade de artigos: a abordagem sobre experiências de inclusão de pacientes surdos relevantes para a prática farmacêutica envolvendo, exclusivamente, farmacêuticos ou estudantes de farmácia.

Como critérios de exclusão estão a abordagem a outras pessoas que não apenas surdas ou deficientes auditivos, por exemplo aqueles que descrevem cuidados farmacêuticos para pessoas surdo-cegas. Considera-se que nesse grupo a comunicação é, normalmente, direcionada aos familiares ou cuidadores. 
Inicialmente, realizou-se a leitura dos títulos dos trabalhos identificados; os manuscritos de interesse foram selecionados para a leitura dos resumos. Após a leitura dos resumos, aqueles relevantes para a investigação foram lidos na íntegra.

\section{Resultados e Discussão}

Dos 499 artigos identificados nas bases de dados consultadas, após a remoção de duplicatas e leitura de títulos e resumo, resultaram em 18 artigos de interesse, desses onze qualificaram para a leitura em profundidade, conforme ilustra a Figura 1. Os artigos incluídos na presente revisão englobaram estudos qualitativos, quali-quantitativos, transversal, simulação realística e baseado em grupo focal. Três estudos descrevem experiências de inclusão para pessoas surdas no contexto da formação profissional de estudantes de Farmácia. Um estudo do tipo transversal contempla a inclusão de ensino de Libras no curso de graduação em Farmácia e sete estudos delineiam exemplos de experiências que visam melhorar a comunicação entre os surdos e os farmacêuticos.

A barreira de comunicação foi identificada como um obstáculo em todos os estudos incluídos nesta revisão. Nesse sentido, diferentes abordagens foram executadas com o propósito de alcançar a inclusão de surdos dentro dos cuidados farmacêuticos, tais como providenciar competência cultural a estudantes de farmácia acerca das características da comunidade surda, prover informações sobre as limitações de pessoas com surdez em comparação com pessoas ouvintes, identificar as necessidades em saúde das pessoas surdas, identificar a estratégia de comunicação mais apropriada conforme o entendimento dos surdos sobre os medicamentos para melhorar a segurança e a efetividade de seu uso.

O Quadro 2 apresenta uma síntese das intervenções de práticas de cuidado realizadas e seus principais resultados no contexto da formação profissional de farmacêuticos e dos profissionais já inseridos nos serviços de saúde. 
Research, Society and Development, v. 11, n. 1, e12411124604, 2022

(CC BY 4.0) | ISSN 2525-3409 | DOI: http://dx.doi.org/10.33448/rsd-v11i1.24604

Figura 1: Fluxograma para a seleção de artigos relevantes na prática de cuidados farmacêuticos para pessoas surdas.

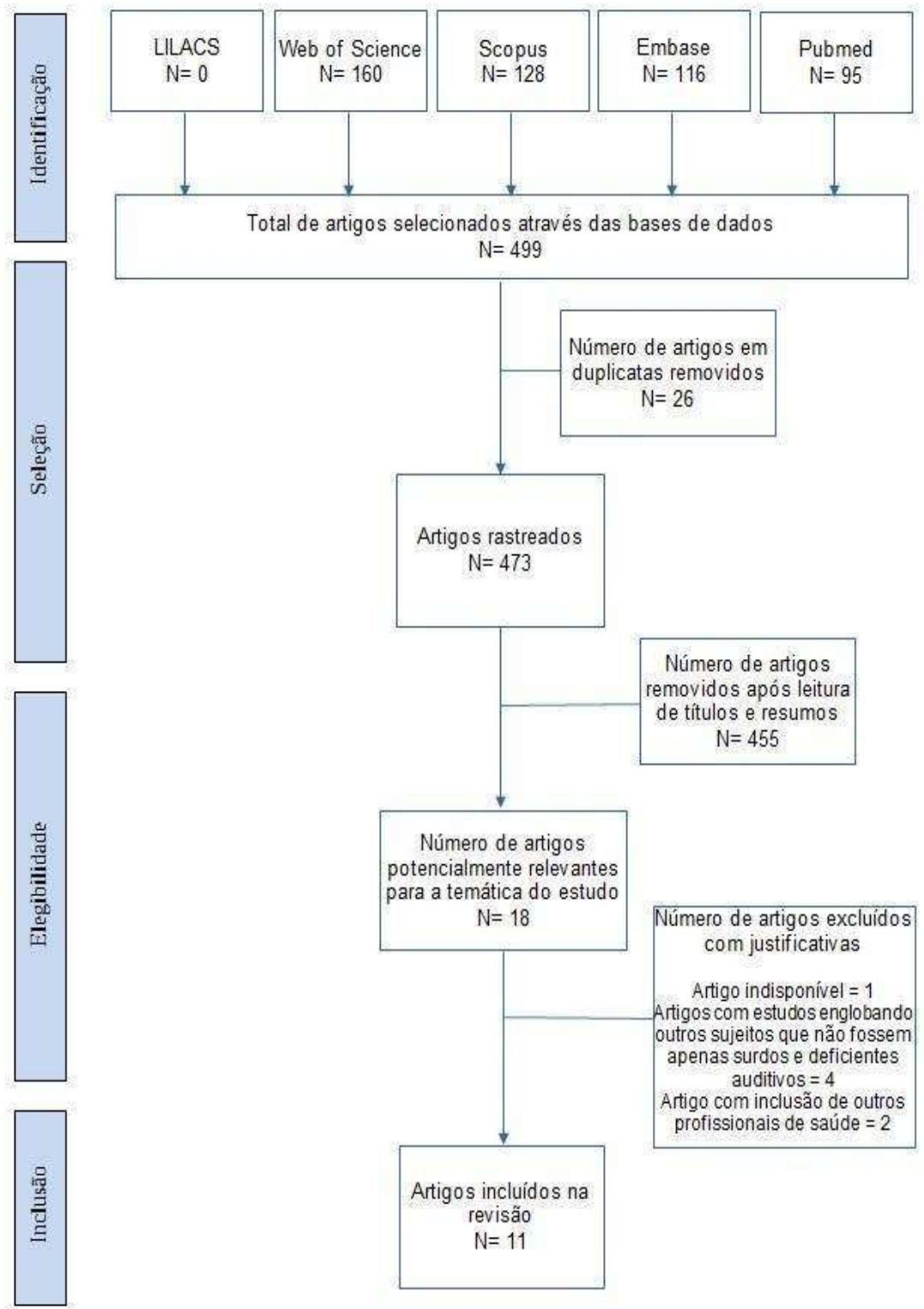

Fonte: Autores. 
Research, Society and Development, v. 11, n. 1, e12411124604, 2022

(CC BY 4.0) | ISSN 2525-3409 | DOI: http://dx.doi.org/10.33448/rsd-v11i1.24604

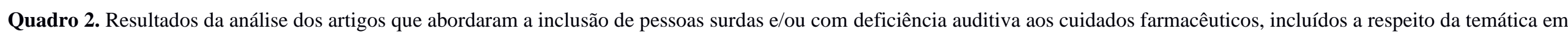
busca nas bases de dados pesquisadas.

\begin{tabular}{|c|c|c|c|c|}
\hline $\begin{array}{l}\text { Autor e ano de } \\
\text { publicação }\end{array}$ & $\begin{array}{c}\text { País de } \\
\text { realização } \\
\text { do estudo }\end{array}$ & Método & $\begin{array}{c}\text { População / } \\
\text { Amostra }\end{array}$ & Principais resultados \\
\hline $\begin{array}{l}\text { Mathews et al., } \\
2011 .\end{array}$ & $\begin{array}{l}\text { Estados } \\
\text { Unidos da } \\
\text { América }\end{array}$ & $\begin{array}{l}\text { Simulação realística em hospital (Deaf Strong } \\
\text { Hospital-DSH) na Escola de Farmácia Wegman do } \\
\text { Colégio John Fischer no primeiro ano do curso de } \\
\text { farmácia em } 2009 \text { como parte do curso obrigatório de } \\
\text { Introdução à Diversidade. Uma sessão de Debriefing } \\
\text { entre os idealizadores e estudantes e uma pesquisa de } \\
\text { opinião sobre a aprendizagem foram utilizadas como } \\
\text { avaliação. }\end{array}$ & $\begin{array}{l}17 \text { facilitadores da } \\
\text { comunidade surda, } \\
\text { oito intérpretes e } \\
76 \text { estudantes de } \\
\text { farmácia. }\end{array}$ & $\begin{array}{l}\text { - Identificar diferenças culturais no contexto de providenciar } \\
\text { cuidados em saúde para pacientes surdos. } \\
\text { - Experiência com a simulação, sendo essa descrita como } \\
\text { educacional e interessante. } \\
\text { - } 97 \% \text { dos alunos concordaram que a experiência alcançou seu } \\
\text { objetivo. }\end{array}$ \\
\hline $\begin{array}{l}\text { Ferguson \& Liu, } \\
2015 .\end{array}$ & $\begin{array}{l}\text { Estados } \\
\text { Unidos da } \\
\text { América }\end{array}$ & $\begin{array}{l}\text { Grupo focal realizado em quatro sessões com duração } \\
\text { média de } 60 \text { minutos, ocorrido entre setembro de } 2013 \\
\text { e abril de } 2014 \text {. As questões do grupo focal foram } \\
\text { abertas e semi-estruturadas baseadas na identificação } \\
\text { de barreiras na comunicação, necessidades de surdos } \\
\text { e deficientes auditivos na requisição de serviços } \\
\text { farmacêuticos e o impacto da qualidade da } \\
\text { comunicação quanto aos erros e adesão de } \\
\text { medicamentos. }\end{array}$ & $\begin{array}{l}16 \text { surdos e quatro } \\
\text { deficientes } \\
\text { auditivos. }\end{array}$ & $\begin{array}{l}\text { - } 35 \% \text { relataram nenhuma tentativa de comunicar com o } \\
\text { farmacêutico no último mês e } 30 \% \text { tentaram se comunicar. } \\
\text { - Uma média de três medicamentos foi prescrita para os pacientes } \\
\text { surdos. } \\
\text { - Foi apontado sobre o não conhecimento do papel e as } \\
\text { responsabilidades de diferentes pessoas que trabalham na } \\
\text { farmácia. } \\
\text { Questões de uso e segurança de medicamentos apontaram pela } \\
\text { falta de instrução. } \\
\text { - Foram reconhecidas uma ampla gama de cuidados em saúde que } \\
\text { farmacêuticos estão aptos a realizar. }\end{array}$ \\
\hline
\end{tabular}




\begin{tabular}{|c|c|c|c|c|}
\hline $\begin{array}{l}\text { Autor e ano de } \\
\text { publicação }\end{array}$ & $\begin{array}{l}\text { País de } \\
\text { realização } \\
\text { do estudo }\end{array}$ & Método & $\begin{array}{c}\text { População / } \\
\text { Amostra }\end{array}$ & Principais resultados \\
\hline $\begin{array}{ll}\text { Ferguson } & \& \\
\text { Shan, 2016. } & \end{array}$ & $\begin{array}{l}\text { Estados } \\
\text { Unidos da } \\
\text { América }\end{array}$ & $\begin{array}{l}\text { Estudo transversal, com aplicação de um questionário } \\
\text { com } 15 \text { itens enviado online ou entregue } \\
\text { pessoalmente, com o objetivo de coletar informações } \\
\text { de farmacêuticos em relação a suas atitudes e métodos } \\
\text { de interação com pacientes surdos. }\end{array}$ & $\begin{array}{l}73 \text { farmacêuticos, } \\
\text { que trabalham em } \\
\text { área com } \\
\text { significativa } \\
\text { população surda. }\end{array}$ & $\begin{array}{l}\text { - 93\%, responderam que eles já interagiram com pacientes surdos. } \\
\text { - } 74 \% \text { responderam ter sido levemente difícil, } 16 \% \text { responderam } \\
\text { não ter sido difícil e } 10 \% \text { acham muito difícil se comunicar com o } \\
\text { surdo. } \\
\text { - } 30 \% \text { dos farmacêuticos responderam que foi algo confortável ou } \\
\text { confortável em providenciar diretamente cuidados para os surdos, } \\
\text { quase } 10 \% \text { se sentiram muito desconfortáveis. } \\
\text { - } 89 \% \text { afirmaram sobre a obrigação legal de garantir uma } \\
\text { comunicação efetiva para os surdos. } \\
\text { - } 79,5 \% \text { apontou que o material escrito foi a principal forma de } \\
\text { providenciar informação para os surdos. }\end{array}$ \\
\hline $\begin{array}{l}\text { Hyoguchi et al., } \\
2016 .\end{array}$ & Japão & $\begin{array}{l}\text { Aplicação de dois questionários, um com } 20 \\
\text { declarações para identificar "certo" e "errado" sobre a } \\
\text { compreensão do uso apropriado de medicamentos e } \\
\text { outro questionário com três questões sobre a atitude } \\
\text { em relação à explicação e uso de medicamentos feitos } \\
\text { pelos farmacêuticos e, a atitude (entendimento, } \\
\text { confiança e segurança) dos participantes a respeito do } \\
\text { uso do medicamento com três itens. Foi providenciada } \\
\text { uma palestra sobre informações básicas de uso de } \\
\text { medicamentos. }\end{array}$ & $\begin{array}{ll}20 \quad \text { surdos, } & 19 \\
\text { deficientes } & \\
\text { auditivos e } & 20 \\
\text { ouvintes. } & \end{array}$ & $\begin{array}{l}\text { - Antes da palestra, os participantes surdos providenciaram uma } \\
\text { média de } 8,5 \text { de respostas corretas }(1-18) \text {, dos participantes } \\
\text { deficientes auditivos menos de } 16 \text { respostas corretas }(11-19) \text { e, } \\
16,5 \text { (15-20) por participantes ouvintes. } \\
\text { - Depois da palestra o número de respostas corretas providenciadas } \\
\text { por surdos e deficientes auditivos aumentou em } 16 \text { e } 19 \text {, } \\
\text { respectivamente. } \\
\text { - Os participantes surdos foram pontuados em grupo com alto } \\
\text { escore e grupo com baixo escore, nove participantes } \\
\text { providenciaram mais de } 16 \text { respostas corretas, que foi o nível de } \\
\text { conhecimento exibido pelos participantes ouvintes. } \\
\text { - Quanto à atitude a respeito do uso do medicamento, após a } \\
\text { palestra, a compreensão dos surdos e deficientes auditivos } \\
\text { aumentaram e a confiança e segurança diminuíram. }\end{array}$ \\
\hline
\end{tabular}




\begin{tabular}{|c|c|c|c|c|}
\hline $\begin{array}{c}\text { Autor e ano de } \\
\text { publicação }\end{array}$ & $\begin{array}{l}\text { País de } \\
\text { realização } \\
\text { do estudo }\end{array}$ & Método & $\begin{array}{c}\text { População / } \\
\text { Amostra }\end{array}$ & Principais resultados \\
\hline $\begin{array}{l}\text { Araújo et al., } \\
2019 .\end{array}$ & Brasil & $\begin{array}{l}\text { Estudo transversal e descritivo que envolve a } \\
\text { avaliação do ensino de Libras quanto às suas } \\
\text { características e frequência dentro da grade curricular } \\
\text { das escolas de graduação em Farmácia de } \\
\text { universidades Federais do Brasil. Realizado no } \\
\text { período de março a junho de 2017, cujas amostras } \\
\text { foram extraídas do site do Instituto Nacional de } \\
\text { Estudos e Pesquisas Educacionais (INEP). }\end{array}$ & $\begin{array}{lr}35 & \text { grades } \\
\text { curriculares } & \text { de } \\
\text { escolas } & \text { de } \\
\text { graduação } & \text { em } \\
\text { Farmácia. } & \end{array}$ & $\begin{array}{l}\text { - 51,4\% oferecem o ensino de Libras como disciplina optativa. } \\
\text { - Uma escola poderia providenciar o curso de Libras com base } \\
\text { teórico-prática, enquanto } 14 \text { cursos oferecem ensino de natureza } \\
\text { apenas teórica. } \\
\text { - } 16 \text { escolas não determinaram um período certo para os estudantes } \\
\text { aprenderem Libras. } \\
\text { - Metade dos currículos analisados possuem } 60 \text { horas de aula. } \\
\text { - O principal conteúdo abordado refere-se ao ensino de Libras e } \\
\text { aprendizado da língua de sinais; } 66,7 \% \text { tratam do tema surdez e } \\
\text { apenas } 22,2 \% \text { abordam sobre questões de políticas públicas e } \\
\text { aspectos de legislação. }\end{array}$ \\
\hline $\begin{array}{l}\text { Chong; } \\
\text { Palanisamy \& } \\
\text { Jacob, } 2019 .\end{array}$ & Malásia & $\begin{array}{l}\text { Estudo qualitativo por discussão de grupo focal. A } \\
\text { validação do conteúdo foi realizada por experts em } \\
\text { pesquisa qualitativa; a sessão teve duração entre uma } \\
\text { a duas horas. }\end{array}$ & $\begin{array}{l}12 \text { farmacêuticos } \\
\text { comunitários. }\end{array}$ & $\begin{array}{l}\text { - Três temas se destacaram pela discussão do grupo focal. } \\
\text { - O desenho do conteúdo do aplicativo mHealth. } \\
\text { - Os benefícios percebidos do aplicativo. } \\
\text { - As questões sobre os desafios potenciais relacionados ao } \\
\text { aplicativo. }\end{array}$ \\
\hline $\begin{array}{l}\text { Hyoguchi; } \\
\text { Ohmitsu \& } \\
\text { Kubota, 2020. }\end{array}$ & Japão & $\begin{array}{l}\text { Uma palestra de } 90 \text { minutos de duração com } \\
\text { abordagem dos seguintes temas: comunidade surda e } \\
\text { deficiente auditiva, dificuldades e ansiedade desses } \\
\text { pacientes relacionadas à comunicação e estratégias de } \\
\text { comunicação. Foi realizada uma simulação de } \\
\text { experiência baseada em perda auditiva de } 90 \text { minutos } \\
\text { de duração, dividida em dois grupos, um de perda } \\
\text { auditiva e outro de ouvintes. E quatro questionários } \\
\text { foram preparados: (I) conhecimento, (II) ações, (III) } \\
\text { confiança e (IV) avaliação do aprendizado. }\end{array}$ & $\begin{array}{l}151 \text { estudantes do } \\
5^{\circ} \text { ano do curso de } \\
\text { Farmácia na } \\
\text { Universidade de } \\
\text { Daiichi }\end{array}$ & $\begin{array}{l}\text { - 23,8\% indicaram conhecimento sobre deficiência auditiva. } \\
\text { - } 39,1 \% \text { destacaram a escrita e } 29,1 \% \text { a língua de sinais, quanto } \\
\text { aos métodos de comunicação. } \\
\text { - } 70 \% \text { dos participantes acreditam que as pessoas não poderiam } \\
\text { realizar leitura labial de forma correta. } \\
\text { - Os participantes afirmaram que falar em voz alta ou falar } \\
\text { próximo ao ouvido são ações que contribuem com a comunicação } \\
\text { com surdos. } \\
\text { - } 132 \text { estudantes indicaram que esse aprendizado contribuiu para } \\
\text { melhorar a confiança deles em interagir com surdos ou deficientes } \\
\text { auditivos e providenciar melhores informações sobre } \\
\text { medicamentos. }\end{array}$ \\
\hline
\end{tabular}




\begin{tabular}{|c|c|c|c|c|}
\hline $\begin{array}{l}\text { Autor e ano de } \\
\text { publicação }\end{array}$ & $\begin{array}{l}\text { País de } \\
\text { realização } \\
\text { do estudo }\end{array}$ & Método & $\begin{array}{c}\text { População / } \\
\text { Amostra }\end{array}$ & Principais resultados \\
\hline $\begin{array}{l}\text { Hyoguchi et al., } \\
2020 .\end{array}$ & Japão & $\begin{array}{l}\text { Aplicação de três questionários com foco em } \\
\text { investigar o conhecimento de farmacêuticos sobre } \\
\text { deficiências auditivas e ações que devem ser } \\
\text { realizadas para eliminar a discriminação contra } \\
\text { pacientes surdos e pacientes com dificuldades } \\
\text { auditivas, englobando avaliação com } 13 \text { itens. E } \\
\text { conhecer sobre as práticas de ações apropriadas e o } \\
\text { nível de confiança de farmacêuticos em providenciar } \\
\text { educação sobre medicamentos com } 15 \text { itens. }\end{array}$ & $\begin{array}{l}216 \text { farmacêuticos } \\
\text { da Yahata } \\
\text { Associação de } \\
\text { farmacêuticos em } \\
\text { Fukuoma. }\end{array}$ & $\begin{array}{l}\text { - 18,9\% afirmaram conhecer a lei de eliminação da discriminação. } \\
\text { - 5,1\% dos participantes possuem conhecimento sobre ações para } \\
\text { eliminação da discriminação. } \\
\text { - 57,4\% dos participantes conheciam sobre a alfabetização de } \\
\text { surdos. } \\
\text { - } 85,6 \% \text { reconhecem que a leitura labial não pode ser traduzida } \\
\text { corretamente. } \\
\text { - Mais da metade dos participantes não praticam ações apropriadas } \\
\text { para pacientes surdos e com deficiência auditiva. } \\
\text { - 35,6\% dos participantes afirmaram ter confiança em providenciar } \\
\text { informações sobre medicamentos. }\end{array}$ \\
\hline $\begin{array}{l}\text { Bayley et al., } \\
2021 .\end{array}$ & $\begin{array}{l}\text { Estados } \\
\text { Unidos da } \\
\text { América }\end{array}$ & $\begin{array}{l}\text { Um curso opcional co-curricular sobre Língua de } \\
\text { Sinais Americano (do inglês ASL) adaptado para } \\
\text { profissionais de Farmácia. Foram ofertadas quatro } \\
\text { aulas de curso de língua de sinais com duração de } 90 \\
\text { minutos cada, seguidas de avaliação pré e pós-curso, } \\
\text { além da administração de um questionário no início e } \\
\text { no final do curso com o objetivo de avaliar o nível de } \\
\text { conforto e confiança na interação de estudantes de } \\
\text { Farmácia e surdos. }\end{array}$ & $\begin{array}{lr}39 \text { estudantes } & \text { do } \\
\text { primeiro } & \mathrm{e} \\
\text { segundo ano } & \text { do } \\
\text { curso } & \text { de } \\
\text { doutorado } & \mathrm{em} \\
\text { Farmácia } & \end{array}$ & $\begin{array}{l}\text { - Os estudantes se sentiram mais informados sobre os surdos e a } \\
\text { comunidade surda, } \\
\text { - Relataram uma melhora no nível de confiança na interação e } \\
\text { comunicação com indivíduos surdos, deficientes auditivos e } \\
\text { intérpretes de ASL. } \\
\text { - Os participantes identificaram a importância em buscar } \\
\text { treinamento em língua de sinais. } \\
\text { - O resultado do questionário apontou que a escrita pode ser um } \\
\text { modo efetivo de se comunicar com surdos e deficientes auditivos } \\
\text { e, eles não concordam que todos os surdos e deficientes auditivos } \\
\text { podem realizar leitura labial. }\end{array}$ \\
\hline $\begin{array}{l}\text { Chong et al., } \\
2021\end{array}$ & Malásia & $\begin{array}{l}\text { Estudo transversal conduzido para farmacêuticos } \\
\text { comunitários de cinco estados de maior concentração } \\
\text { desses profissionais na península da Malásia; no } \\
\text { período de julho de } 2016 \text { a dezembro de } 2016 \text {. Um } \\
\text { questionário autoaplicável foi desenvolvido baseado } \\
\text { na avaliação do nível de conforto de farmacêuticos } \\
\text { comunitários quando interagem com surdos e/ou } \\
\text { pacientes com deficiência auditiva. A pesquisa }\end{array}$ & $\begin{array}{l}297 \text { farmacêuticos } \\
\text { comunitários }\end{array}$ & $\begin{array}{l}\text { - 63\%, receberam menos de cinco prescrições diárias, sendo que o } \\
\text { número de prescrições diárias teve um significativo impacto no } \\
\text { nível de conforto desses profissionais. } \\
\text { - Experiências prévias com esses usuários se associaram com alto } \\
\text { nível de conforto dos farmacêuticos quando comparado com } \\
\text { aqueles que nunca interagiram com surdos. } \\
\text { - Nenhuma diferença significativa foi associada entre o nível de } \\
\text { conforto e os anos de experiência. } \\
\text { - A maioria dos profissionais usa a informação por escrito e } \\
\text { também a maioria preferem esse tipo de comunicação seguido de }\end{array}$ \\
\hline
\end{tabular}


Research, Society and Development, v. 11, n. 1, e12411124604, 2022

(CC BY 4.0) | ISSN 2525-3409 | DOI: http://dx.doi.org/10.33448/rsd-v11i1.24604

\begin{tabular}{|c|c|c|c|c|}
\hline $\begin{array}{l}\text { Autor e ano de } \\
\text { publicação }\end{array}$ & $\begin{array}{l}\text { País de } \\
\text { realização } \\
\text { do estudo }\end{array}$ & Método & $\begin{array}{c}\text { População / } \\
\text { Amostra }\end{array}$ & Principais resultados \\
\hline & & $\begin{array}{l}\text { constituiu-se em } 14 \text { itens fechados subdivididos em } \\
\text { cinco sessões. }\end{array}$ & & $\begin{array}{l}\text { um acompanhante intérprete ou uso da língua de sinais, porém a } \\
\text { comunicação escrita não teve relação com o nível de conforto dos } \\
\text { farmacêticos. }\end{array}$ \\
\hline $\begin{array}{l}\text { Jacob et al., } \\
2021\end{array}$ & Malásia & $\begin{array}{l}\text { Dois grupos de discussão focal. O roteiro de entrevista } \\
\text { foi desenvolvido com base na visão de surdos sobre a } \\
\text { proposta do aplicativo mHealth em termos de desenho } \\
\text { e características, orientados por especialistas em } \\
\text { pesquisa qualitativa, membros da comunidade surda e } \\
\text { especialistas em desenvolvimento e design de } \\
\text { aplicativos. }\end{array}$ & $\begin{array}{lr}10 \quad \text { surdos } & \text { ou } \\
\text { pessoas } & \text { com } \\
\text { dificuldades } & \\
\text { auditivas. }\end{array}$ & $\begin{array}{l}\text { - Foram gerados dois temas: (I) desafios e ceticismo em relação ao } \\
\text { sistema de cuidados em saúde e (II) características do aplicativo } \\
\text { mHealth. } \\
\text { - Sete participantes concordaram que a comunicação com } \\
\text { profissionais farmacêuticos é um desafio. } \\
\text { - Seis participantes reconheceram que existe uma parcela de surdos } \\
\text { que não são alfabetizados ou não poderiam compreender } \\
\text { totalmente informações por escrito. } \\
\text { - Os participantes preferem vídeos acompanhados de subtítulos, } \\
\text { com sugestão para vários idiomas e fotos relacionadas aos } \\
\text { medicamentos. } \\
\text { - O consenso foi que o aplicativo deveria ser disponibilizado de } \\
\text { forma gratuita. }\end{array}$ \\
\hline
\end{tabular}

Fonte: Autores. 
Chong, Palanisamy e Jacob (2019) apontam que muitos profissionais de saúde se sentem despreparados para providenciar melhores cuidados aos pacientes surdos, devido à falta de conhecimento sobre a cultura surda e seus direitos. Para entender as necessidades de surdos, é preciso conhecer o ambiente cultural em que a pessoa está inserida, uma vez que os fatores culturais influenciam no processo saúde-doença (Chong, Palanisamy \& Jacob, 2019); logo, é importante considerar os aspectos de diversidade cultural na formação de profissionais de saúde (Ferguson \& Liu, 2015). No Brasil, o decreto n ${ }^{\circ} 5.626$, de 22 de dezembro de 2005, dispõe sobre a garantia do direito à saúde para surdos e pessoas com deficiência auditiva, efetivando, através deste, dentre outras ações, a de capacitação e formação de profissionais da Rede de Atenção em Saúde do Sistema Único Saúde (SUS), a promoção do uso de Libras, a sua tradução e interpretação (Brasil, 2005).

A legislação pode contribuir para que os trabalhadores inseridos nos serviços de saúde adquiram o entendimento de deficiência auditiva, bem como da surdez. No entanto, as ações para a promoção da inclusão de surdos nos cuidados em saúde passam pela conscientização e eliminação da discriminação entre os profissionais de saúde (Hyoguchi et al., 2020). Ademais, as experiências de aprendizagem a partir da interação com os demais atores dos serviços, com a comunidade e baseado nas práticas farmacêuticas correntes, são fundamentais para auxiliar melhores desfechos em saúde e promover a autonomia dos pacientes (Vinholes, Alano \& Galato, 2009).

As abordagens utilizadas por iniciativas universitárias para promover o conhecimento de Língua de Sinais destacaram a importância da educação continuada, das metodologias em comunicação e/ou das observações clínicas e avaliações subsequentes do aprendizado para melhorar as habilidades linguísticas dos estudantes de Farmácia (Bailey et al., 2021). Em adição, tais métodos podem contribuir para melhorar o nível de confiança dos estudantes e profissionais quanto à informação sobre medicamentos aos surdos ou deficientes auditivos (Hyoguchi et al., 2020).

No Brasil, a inclusão da Libras como disciplina curricular optativa nos cursos de educação profissional está embasada pelo decreto $\mathrm{n}^{\circ} 5626$, de 2005. A oferta de um curso de língua de sinais na grade curricular do ensino de farmácia mostra a necessidade de discutir sobre a cultura surda, os aspectos histórico, social, antropológico e clínico, com a finalidade de providenciar um cuidado humanista e integral para pacientes surdos (Araújo et al., 2019).

Muitos cursos de Farmácia ainda não incluem em suas grades curriculares cuidados e serviços farmacêuticos para surdos ou deficientes auditivos (Bailey et al., 2021). Araújo e colaboradores (2019) apresentaram uma análise sobre a incorporação do ensino de Libras nos cursos de graduação de Farmácia em universidades federais, identificaram que o conteúdo programático dessa disciplina não reflete as reais demandas de pacientes surdos quando estes buscam os serviços farmacêuticos evidenciando as lacunas ao que concerne a criação de conteúdos curriculares para abranger os cuidados em saúde de surdos (Araújo et al., 2019).

Existem surdos que não possuem bom domínio da linguagem escrita, o que pode resultar em dificuldades em compreender materiais escritos, como cartazes, folhetos ou artigos (Hyoguchi et al., 2016). Além disso, os termos médicos específicos muitas vezes não são entendidos pelos pacientes e eles se sentem desconfortáveis em questionar sobre as dúvidas, o que pode trazer resultados negativos para o tratamento (Mathews et al., 2011). Sendo que, o baixo nível de compreensão em leitura e o pobre conhecimento de assuntos sobre saúde são mais prevalentes em pessoas surdas do que em ouvintes (Hyoguchi, Ohmitsu \& Kubota, 2020).

Para informar sobre medicamentos às pessoas surdas, é necessário que os farmacêuticos tenham habilidades de realizar uma comunicação efetiva com esses usuários (Hyoguchi, Ohmitsu \& Kubota, 2020). E, assim, assegurar que eles possam compreender sobre o uso de medicamentos de acordo com suas condições clínicas, assim como providenciar os cuidados em saúde de forma efetiva e segura (Vieira, 2006; Ferguson \& Shan, 2016). Abordagens que podem auxiliar a comunicação entre surdos e profissionais de saúde têm sido difundidas (NAD, 2021). A utilização de aplicativos para saúde engloba desfechos 
positivos para melhorar a adesão ao tratamento, por exemplo, é importante para controlar determinadas doenças ou favorecer a sua curabilidade (Chong, Palanisamy \& Jacob, 2021).

Chong; Palanisamy e Jacob (2019) apresentam o aplicativo mHealth que visa melhorar a comunicação entre farmacêuticos e surdos o qual deve conter funções que facilitem o conhecimento de surdos acerca de sua farmacoterapia, apostar na dinamicidade e interatividade além de conter vídeos com subtítulo em língua de sinais. Ademais, outros recursos podem ser inseridos como informação sobre medicamentos e história medicamentosa, resultados de exames laboratoriais e histórico de alergias. É importante ressaltar que a disponibilidade do aplicativo não visa substituir as consultas farmacêuticas aos usuários que buscam cuidados farmacêuticos (Chong, Palanisamy \& Jacob, 2021).

Os estudos que descreveram serviços farmacêuticos para pacientes surdos nos diversos cenários de cuidado investigaram quais são os conhecimentos desses profissionais acerca dos métodos de comunicação, a obrigação legal quando as informações sobre medicamentos são complexas e, nesse contexto, os farmacêuticos deveriam providenciar a presença de um intérprete para garantir a segurança quanto ao uso do medicamento (Ferguson \& Shan, 2016; Hyoguchi et al., 2020). As obrigações legais dos serviços farmacêuticos relacionados ao cuidado centrado no paciente, servindo em suas necessidades e otimizando a qualidade de vida do paciente, mostram que ainda existem lacunas sobre as melhores práticas de cuidado baseadas em evidências para pessoas surdas (Ferguson \& Shan, 2016).

A maioria dos estudos traz os métodos de comunicação para a interação entre farmacêuticos e surdos (Chong et al., 2021). A comunicação por escrita é o método mais utilizado; entretanto, não se pode afirmar que surdos compreendem o que os farmacêuticos informam sobre medicamentos por causa do desconhecimento por parte dos farmacêuticos acerca da alfabetização dos surdos, como reportado por Ferguson e Shan (2016). Alguns farmacêuticos preferem se comunicar com surdos por leitura labial; entretanto, existem palavras homônimas e, nesse contexto, os farmacêuticos não conseguem determinar se os surdos podem compreender as peculiaridades na tomada de alguns medicamentos, como tomar em dias alternados, alternar as concentrações, tomar em horário fracionado durante o dia (Hyoguchi et al., 2020).

De acordo com a Organização Mundial da Saúde (OMS), os farmacêuticos comunitários são profissionais de saúde acessíveis (WHO, 1997; Chong et al., 2021); portanto, é relevante na prática desse profissional considerar uma postura acolhedora, que respeite a individualidade e favoreça a troca de informações com indivíduos surdos, principalmente na atenção primária (Chong, Palanisamy \& Jacob, 2019; NAD, 2021).

Para promover uma assistência terapêutica integral e a qualidade do cuidado é sabido que a provisão de medicamentos não é o único serviço a ser ofertado por farmacêuticos (Ilardo \& Speciale, 2020), assim, esses profissionais devem conhecer as demandas da sociedade em especial de pessoas vulneráveis, tais como as que apresentam deficiências (Ferguson \& Liu, 2015). Outras faces de atenção em saúde que necessitam ser resgatadas são: as crenças e cultura de cada indivíduo, o ambiente físico e social que influenciam nas respostas do processo saúde-doença-cuidado (Soares et al.,2016; Ilardo \& Speciale, 2020).

O profissional farmacêutico pode cooperar assim com o seu papel na prevenção da doença, promoção e recuperação da saúde desta população (Soares et al., 2016). Da mesma forma observa-se o potencial protagonista de farmacêuticos nas intervenções que previnem a perda auditiva relacionada ao uso de medicamentos.

As limitações no presente trabalho foram não incluir intervenções farmacêuticas abordadas em outros idiomas, além do português, inglês e espanhol e a impossibilidade de obter artigos para leitura na íntegra, dentre os quais um estudo transversal que expõe sobre a promoção do uso racional de medicamentos pela comunidade surda no Brasil. 


\section{Conclusão}

A revisão integrativa demonstrou que existem estudos relevantes sobre as práticas de cuidados farmacêuticos que visam a mitigar os obstáculos de comunicação entre profissionais farmacêuticos e surdos. O treinamento de estudantes de graduação em farmácia no âmbito dos cuidados farmacêuticos para a inclusão de surdos demonstra resultados positivos de melhora de confiança e de conhecimento sobre cultura surda e promoção de abordagens que produzam comunicação em saúde de qualidade. É evidente que semelhantes métodos podem ser difundidos nos diversos cenários de atenção à saúde, tendo em vista que a responsabilidade de profissionais de saúde, inclusive dos farmacêuticos, em providenciar educação sobre medicamentos de forma segura e promover saúde, também se encontra embasada na legislação brasileira. Esperamos que a difusão de trabalhos presentes nessa revisão integrativa possa ser um gatilho para que os diversos autores que atuam na assistência em saúde para a sociedade possam incluir pessoas com surdez na oferta de serviços em saúde, dentre as quais a atenção oncológica poderia ser uma prática importante.

\section{Referências}

Araújo, D. C. S. A et al. (2019). Sign Language in Brazilian Pharmacy Education. American Journal of Pharmaceutical Education, 83(8), 1676-1680. DOI:10.5688/ajpe7239.

Bailey, N. et al. Evaluation of an American Sign Language co-curricular training for pharmacy students. (2021). Currents in Pharmacy Teaching and Learning, 13(1), 68-72. DOI: 10.1016/j.cptl.2020.08.002.

Instituto Brasileiro de Geografia e Estatística. Estatísticas de Gênero (IBGE). (2021). https://www.ibge.gov.br/apps/snig/v1/?loc=0\&cat=-1,-2,-3,128\&ind=4643.

Brasil. (2002). Lei n ${ }^{\circ} 10.436$ de 24 de abril de 2002. Dispõe sobre a Língua Brasileira de Sinais- Libras e dá outras providências. Diário Oficial [da] República Federativa do Brasil: Seção 1, Brasília. DF, p. 23.

Brasil. (2005). Decreto ${ }^{\circ} 5.626$ de 22 de dezembro de 2005. Regulamenta a Lei $\mathrm{n}^{\circ}$ 10.436, de 24 de abril de 2002, que dispõe sobre a Língua Brasileira de Sinais - Libras, e o art. 18 da Lei n ${ }^{\circ}$ 10.098, de 19 de dezembro de 2000. Diário Oficial [da] República Federativa do Brasil: Seção 1, Brasília. DF, p. 28.

Chong, E. Y-C.; Palanisamy, U. D. \& Jacob, S. A. (2019). A qualitative study on the design and development of an mHealth app to facilitate communication with the Deaf community: perspective of community pharmacists. Patient Preference and Adherence, 13, 195-207. DOI:10.2147/PPA.S182516.

Chong E.Y. et al. (2021). Assessment of community pharmacists' communication and comfort levels when interacting with Deaf and hard of hearing patients. Pharmaceutical Practice, 19(2). DOI:10.18549/PharmPract.2021.2.2274.

Ferguson, McK. \& Liu, M. (2015). Communication needs of patients with altered hearing ability: Informing pharmacists patient care services through focus groups. Journal of the American Pharmacists Association, 5(2), 153-160. DOI:10.1331/JAPhA.2015.14147.

Ferguson, McK. \& Shan, L. (2016). Survey Evaluation of Pharmacy Practice Involving Deaf Patients. Journal of Pharmacy Practice, 29(5), 461-466. DOI: $10.1177 / 0897190014568379$.

Hyoguchi, N. et al. (2016). Effects on Deaf Patients of Medication Education by Pharmacists. The Journal of Deaf Studies and Deaf Education, 21(4), 416-421. DOI:10.1093/deafed/enw037.

Hyoguchi, N., Ohmitsu, M. \& Kubota, T. (2020). Effects of Learning on the Confidence to Provide Medication Education for Pharmacy Students. Yakugaku Zasshi, 140(6), 827-838. DOI: 10.1248/yakushi.19-00232.

Hyoguchi, N. et al. (2020). Survey of Pharmacists' Knowledge, Actions and Confidence in Medication Education to Patients with Hearing Disabilities. Yakugaku Zasshi, 140(10), 1295-1298. DOI: 10.1248/yakushi.20-00100.

Ilardo, M. L. \& Speciale, A. (2020). The Community Pharmacist: Perceived Barriers and Patient-Centered Care Communication. International Journal of Environmental Research and Public Health, 17(536). DOI: 10.3390/ijerph17020536.

Jacob S.A. et al. (2021). Design suggestions for an mHealth app to facilitate communication between pharmacists and the Deaf: perspective of the Deaf community (HEARD Project). Mhealth, 7(29). DOI: 10.21037/mhealth.2020.01.04.

Mathews, J. L. et al. (2011). Role-Reversal Exercise with Deaf Strong Hospital to Teach Communication Competency and Cultural Awareness. American Journal of Pharmaceutical Education, 75(3). DOI: 10.5688/ajpe75353.

National Association of the Deaf (NAD). (2021). Position Statement on Health Care Access for Deaf Patients. Estados Unidos. https://www.nad.org/aboutus/position-statements/position-statement-on-health-care-access-for-deaf-patients/.

Soares, L. et al. (2016). Assistência Farmacêutica no Brasil: Política, Gestão e Clínica. Atuação clínica do farmacêutico. Florianópolis. Ed. da UFSC, 5 , 353 p. 
Research, Society and Development, v. 11, n. 1, e12411124604, 2022

(CC BY 4.0) | ISSN 2525-3409 | DOI: http://dx.doi.org/10.33448/rsd-v11i1.24604

Vieira, F. S. (2006). Possibilidades de contribuição do farmacêutico para a promoção da saúde. Ciência \& Saúde Coletiva. https://www.cienciaesaudecoletiva.com.br/artigos/possibilidades-de-contribuicao-do-farmaceutico-para-a-promocao-da-saude/52?id=52\&id=52\&id=52.

Vinholes, E. R.; Alano, G. M. \& Galato, D. (2009). A percepção da comunidade sobre a atuação do Serviço de Atenção Farmacêutica em ações de educação em saúde relacionadas à promoção do uso racional de medicamentos. Saúde e Sociedade, 18(2), 293-303. DOI:10.1590/S0104-12902009000200012.

Whittemore, R. \& Knafl, K. (2005). The integrative review: update methodology. Journal of Advanced Nursing, 52(5), 546-553. DOI:10.1111/j.13652648.2005.03621.x.

WORLD HEALTH ORGANIZATION (WHO). (2021) Deafness and Hearing loss. https://www.who.int/news-room/fact-sheets/detail/deafness-and-hearingloss.

WORLD HEALTH ORGANIZATION (WHO). (1997) The Role of the Pharmacist in the Health-Care System - Preparing the Future Pharmacist: Curricular Development Report of a Third WHO Consultative Group on the Role of the Pharmacist. Vancouver. https://apps.who.int/iris/bitstream/handle/10665/63817/WHO_PHARM_97_599.pdf?sequence=1\&isAllowed=y. 